\title{
Micro-local analysis of operators \\ WITH NON-INVOLUTIVE CHARACTERISTICS
}

\section{Baliganahalli Raja Iyengar Nagaraj}

The aim of the thesis is to study certain classes of partial and pseudo-differential operators employing micro-local techniques. For this purpose the thesis begins with a comprehensive description of the theory of wave front sets due to $\mathrm{L}$. Hormander [2]. A byproduct of the derivation of these tools is the following result which is of independent interest: If $x \in C^{\infty}\left(\mathbb{R}^{n}\right)$ is of order $m$ at $\infty$ and derivatives of order $|\alpha|$ are $O(m-|\alpha|)$ at $\infty$ then the so called Friedrichs operator $\chi(D)$ is defined by $x(D) V(X)=(2 \pi)^{-n} \int x(\xi) \hat{V}(\xi) e^{i x \xi} d \xi$ where $\hat{V}$ is the Fourier transform of $V \in E^{\prime}$, the space of distributions with compact support; then $f \in O_{c}^{\prime}$ is in $S$ if and only if there exists a Friedrichs operator $X(D)$ such that $f=x(D) V$ for some $V \in E^{\prime}$.

The thesis develops a unified view of the reduction theory of partial differential or more generally pseudo-differential equations leading to the interpretation of the Egorov-Maslov reduction as a $C^{\infty}$ equivalence theorem under local homogeneous symplectic deformations of the cotangent bundle which are consistent with equivalence under local diffeomorphisms of the base provided they define canonical deformations.

on a $C^{\infty}$ manifold $X$ we study the class of pseudo-differential

Received 5 February 1986. Thesis submitted to University of Western Australia, December 1982. Degree approved November 1985. Supervisor: Professor J.P.O. Silberstein.

Copyright Clearance Centre, Inc. Serial-fee code: 0004-9727/87 $\$ A 2.00+0.00$. 
operators

$$
R=P Q^{2}+A Q+B
$$

where $P, Q$ are pseudo-differential operators with real principal symbols of order 0 and 1 respectively and $A, B$ are operators of order 0 . In addition we assume that the characteristic sets of $P$ and $Q$ have non-involutive intersection, in other words the Poisson bracket of principal symbols $\sigma(P)$ and $\sigma(Q)$ of $P$ and $Q$ respectively satisfies the condition

$$
\{\sigma(P), \sigma(Q))\}\left(z_{0}\right) \neq 0 \text { whenever } \sigma(P)\left(z_{0}\right)=0 \text { and } \sigma(Q)\left(z_{0}\right)=0 .
$$

It is shown that $R$ is micro-locally equivalent in a conic neighbourhood of its non-involutive triple characteristics to the class of operators

$$
R^{\prime}=t D_{t}^{2}+A_{0} D_{t}+B_{0}
$$

where $A_{0}, B_{0}$ are operators of order 0 . Using $(x, t, \xi, \tau)$ as local coordinates in $T^{*}(X)$ the operator has $\sigma\left(R^{\prime}\right)=t \tau^{2}$ near $t=\tau=0$.

Micro-local parametrices are constructed for operators of class $R^{\prime}$ when $A_{0}$ and $B_{0}$ are complex constants. This case is the simplest but still has all the essential features. The parametrices are used to obtain information on the propagation of singularities and it is found that the results obtained closely resemble those of Hanges [1] and Ivrii [3] for the case of non-involutive double characteristics except in the case when $A_{0}$ is an integer, when extra conditions are required to obtain propagation results of the type given in Hanges [1] Theorem 1.

The operator $R^{\prime}$ with $A_{0}, B_{0} \in \mathbb{C}$ is also micro-locally equivalent to a third order partial differential operator on $\mathbb{I R}^{2}$ of the form

$$
N\left(A_{0}, B_{0}\right)=\left(D_{x}-(x-1) D_{y}\right)\left(D_{x}+D_{y}\right)^{2}+A_{0}\left(D_{x}+D_{y}\right) D_{y}+B_{0} D_{y} .
$$

To clarify the behaviour of operators of this type we study the Cauchy problem on $\mathbb{R}^{2}$ of

$$
\left(D_{x}+D_{y}\right)\left(D_{x}-(x-1) D_{y}\right) \text { and }\left(D_{x}-D_{y}\right)\left(D_{x}+(x-1) D_{y}\right)
$$

which have non-involutive double characteristics on the line $x=0$. 
We consider the initial data to be distributions on $x=0$ and analyse the solution from the point of view of reflection on the boundary. It is shown that the Cauchy problem is well posed and that singularities. are propagated along the null-bicharacteristics of the individual factors starting from the ouble characteristics $\left(0, y_{0}, \pm n_{0}, n_{0}\right)$.

\section{References}

[1] N. Hanges, "Propagation of Singularities for a class of operators with double characteristics", Ann. of Math. Stud. Princeton 91 (1979), 113-126.

[2] L. Hormander, "Linear differential operators", Actes Cong. Int. Math. 1 (1970), 121-133.

[3] V. Ya. Ivrii, "Wave fronts of solutions of certain pseudodifferential equations", Functional Anal. Appl. 10 (1976). $141-142$.

[4] B.R. Nagaraj, "A characterization in the space of convolution operators", Proc. Amer. Math. Soc. 94 (1985), 396-398.

Tata Institute of Fundamental Research,

Post Box No. 1234,

Bangalore 560012 ,

India. 